\title{
Heavy lepton pair production in nucleus-nucleus collisions at LHC energy - a case study
}

\author{
Jan-e Alam, Bedangadas Mohanty \\ Variable Energy Cyclotron Center, 1/AF, Bidhan Nagar, Kolkata-700064 \\ Sanjay K Ghosh, Sarbani Majumder, Rajarshi Ray \\ Center for Astroparticle Physics 83 Space Science, \\ Block-EN, Sector-V, Salt Lake, \\ Kolkata-700091, INDIA \\ E \\ Department of Physics, Bose Institute, \\ 93/1, A. P. C Road, Kolkata - 700009, INDIA
}

(Dated: November 7, 2018)

\begin{abstract}
We present a study of $\tau^{+} \tau^{-}$lepton pair production in $\mathrm{Pb}+\mathrm{Pb}$ collisions at $\sqrt{s_{N N}}=5.5 \mathrm{TeV}$. The larger $\tau^{ \pm}$mass $(\sim 1.77 \mathrm{GeV})$ compared to $e^{ \pm}$and $\mu^{ \pm}$leads to considerably small hadronic contribution to the $\tau^{+} \tau^{-}$pair invariant mass $(M)$ distribution relative to the production from thermal partonic sources. The quark-anti-quark annihilation processes via intermediary virtual photon, $\mathrm{Z}$ and Higgs bosons have been considered for the production of $\tau^{+} \tau^{-}$. We observe that the contribution from Drell-Yan process dominates over thermal yield for $\tau^{+} \tau^{-}$pair mass from 4 to $20 \mathrm{GeV}$ at the LHC energy. We also present the ratio of $\tau$ lepton pair yields for nucleus-nucleus collisions relative to yields from $\mathrm{p}+\mathrm{p}$ collisions scaled by number of binary collisions at LHC energies as a function $\tau$ pair mass. The ratio is found to be significantly above unity for the mass range 4 to $6 \mathrm{GeV}$. This indicates the possibility of detecting $\tau^{+} \tau^{-}$pair from quark gluon plasma (QGP) in the mass window $4 \leq M(\mathrm{GeV}) \leq 6$.
\end{abstract}

PACS numbers: 25.75.Ld

\section{INTRODUCTION}

Dilepton production in high energy heavy-ion collisions have been shown to be an excellent observable for studying various dynamical aspects of the evolution of the system formed in heavy-ion collisions 1 3] (see 44 for a review). The lepton pair mass distribution has been used for the diagnostics of QGP formation as well as to study the in-medium properties of low mass vector mesons [5-7]. The transverse momentum $\left(p_{\mathrm{T}}\right)$ distribution of lepton pairs in various $M$ ranges has been used to study the systems radial flow development [8]. The HBT interferometry using dilepton pairs has been proposed to provide information on the time development of collectivity in heavy-ion collisions [9].

Measurements at SPS (center of mass energy, $\left.\sqrt{s_{N N}}=17.3 \mathrm{GeV}\right)[10,11]$ and RHIC $\left(\sqrt{s_{N N}}=200\right.$ $\mathrm{GeV}$ ) [12] have provided results for $e^{+} e^{-}$and $\mu^{+} \mu^{-}$ lepton pairs. Hence theoretical calculations have so far also concentrated on $e^{+} e^{-}$and $\mu^{+} \mu^{-}$dilepton production. With the starting of the heavy-ion collision program at Large Hadron Collider (LHC) a value of $\sqrt{s_{\mathrm{NN}}}=2.76 \mathrm{TeV}$ has been achieved for $\mathrm{Pb}+\mathrm{Pb}$ collisions and and it is planned to reach $\sqrt{s_{N N}}=5.5 \mathrm{TeV}$ shortly. At the LHC energies we expect significant production of the third generation lepton, the $\tau$-leptons. This opens up the possibility to study $\tau^{+} \tau^{-}$pair production in addition to $e^{+} e^{-}$ and $\mu^{+} \mu^{-}$pairs at these energies. The major advantage of looking at $\tau^{+} \tau^{-}$lepton pair arises due to the mass of the $\tau(\sim 1.77 \mathrm{GeV})$. The $\tau$ pair mass distribution would then start beyond the low mass hadronic resonances $(\omega, \rho$ and $\phi)$. That is $\rho, \omega$ and $\phi$ can not "pollute" (unlike $e^{+} e^{-}$or $\mu^{+} \mu^{-}$) the $\tau^{+} \tau^{-}$ contributions from QGP in this mass domain, making it easier to detect QGP through their measurement. The $\tau^{+} \tau^{-}$pair production from the decays of heavy flavours is expected to be small in the mass region around $4 \mathrm{GeV}$. This would in turn mean the remaining contribution for $\tau$ production are due to thermal partonic sources and the Drell Yan (DY). Although the yield of $\tau^{+} \tau^{-}$will be lower compared to other lepton pairs, the yield at LHC should be significant enough to make their detection possible.

In this paper we present a case study of heavy lepton pair production for central $\mathrm{Pb}+\mathrm{Pb}$ collisions at mid-rapidity for $\sqrt{s_{N N}}=5.5 \mathrm{TeV}$. In section II we discuss various processes for $\tau^{+} \tau^{-}$production. Section III is dedicated for the space-time description of the evolving thermal medium formed in the collision. Results for thermal as well as DY production are presented in section IV. Finally we summarize our results on this case study in section $\mathrm{V}$. 


\section{SOURCE OF $\tau$ DILEPTON PAIR PRODUCTION}

The main processes for $\tau^{ \pm}$pair production is by quark and anti-quark annihilation via intermediary photon, $\mathrm{Z}$ and Higgs bosons. The production of $\tau^{+} \tau^{-}$is significant for $M \sim M_{Z}$ and $M \sim M_{H}$ for processes mediated by $Z$ and Higgs bosons respectively, for lower $M$ the contributions from these processes are negligible. The corresponding Feynman diagrams are shown in Fig. 1] They all contribute to thermal production of $\tau^{ \pm}$pair in quark gluon plasma, in principle as well as in the DY process.

The productions for these processes are evaluated from the matrix elements indicated below. The matrix element for the process $q \bar{q} \rightarrow \tau^{+} \tau^{-}$via $Z$ is given by,

$M_{Z}=\frac{g^{2}}{4 \cos ^{2} \theta_{w}} \frac{1}{\left(q^{2}-m_{z}^{2}\right)}\left[v\left(\bar{p}_{2}\right) \Gamma_{q} u\left(p_{1}\right)\right]\left[u\left(\bar{k}_{1}\right) \Gamma_{\tau} v\left(k_{2}\right)\right]$

with

$$
\Gamma_{q}=\gamma^{\mu}\left(c_{V}^{q}-c_{A}^{q} \gamma_{5}\right)
$$

and

$$
\Gamma_{\tau}=\left[\gamma_{\mu}-\frac{q_{\mu} \gamma_{\nu} q^{\nu}}{m_{z}{ }^{2}}\right]\left[c_{V}^{\tau}-c_{A}^{\tau} \gamma_{5}\right]
$$

where $\theta$ is the weak mixing angle, $g$ is weak coupling strength, $c_{V}$ 's and $c_{A}$ 's are vectors and axial vector couplings [13]. The matrix element for the photon mediated process is given by:

$$
M_{\gamma}=\frac{e_{q} e}{q^{2}}\left[v\left(\bar{p}_{2}\right) \gamma^{\mu} u\left(p_{1}\right)\right]\left[u\left(\bar{k}_{1}\right) \gamma_{\mu} v\left(k_{2}\right)\right]
$$

$e_{q}$ is the average charge of quarks, e is the electronic charge. Finally, the matrix element for the Higgs mediated process is:

$$
M_{H}=\frac{m_{q} m_{\tau}}{v^{2}\left(s-m_{H}^{2}\right)}\left[v\left(\bar{p}_{2}\right) u\left(p_{1}\right)\right]\left[u\left(\bar{k}_{1}\right) v\left(k_{2}\right)\right]
$$

where $\left(p_{1}, p_{2}\right)$ and $\left(k_{1}, k_{2}\right)$ are initial state and final state momenta respectively and $v(\sim 246 \mathrm{GeV}[13])$ is the vacuum expectation value of Higgs field. $m_{q}$, $m_{\tau}, m_{Z}, m_{H}$, are the masses of quarks, $\tau$ leptons, $Z$ boson and Higgs respectively. The total production cross section $\left(\sigma_{q}\right)$ of $\tau^{+} \tau^{-}$is obtained by taking a coherent sum of the matrix elements given in Eqs. 1, 2 and 3 with the following values of various parameters: $M_{\tau}=1.78 \mathrm{GeV}, m_{Z}=91 \mathrm{GeV}$, $m_{H}=120 \mathrm{GeV}, \sin \theta_{w}=0.234, c_{A}^{q}=0.5, c_{V}^{q}=0.19$, $c_{A}^{\tau}=-0.5$ and $c_{V}^{\tau}=-0.03$.

The production of DY pair from $p p$ collision is obtained by folding the partonic cross section $\left(\sigma_{q}\right)$ by the parton distribution functions (PDF) as follows [14]

$$
\begin{aligned}
\frac{d \sigma^{p p}}{d M^{2} d y}= & \frac{1}{N_{c}} \frac{d x_{1}}{x_{1}} \int \frac{d x_{2}}{x_{2}} \sum_{q} \sigma_{q}\left[q\left(x_{1}\right) \bar{q}\left(x_{2}\right)+(1 \leftrightarrow 2)\right] \\
& \times \delta\left(y-\frac{1}{2} \ln \frac{x_{1}}{x_{2}}\right)
\end{aligned}
$$

where $\sigma_{q}$ is the partonic cross section, $q\left(x_{i}\right)\left(\bar{q}\left(x_{i}\right)\right)$ is the quark (anti-quark) distribution in nucleon, $x_{i}$ is the Bjorken scaling variable, $y$ is the rapidity and $N_{c}$ is the number of colours. In the present work CTEQ5M PDF's [15] have been taken for the evaluation of the DY contributions. The dilepton yield from leading order $\mathrm{DY}$ process in $\mathrm{Pb}+\mathrm{Pb}$ collisions is obtained as follows:

$$
\frac{d N}{d M^{2} d y}=\frac{N_{\mathrm{coll}}(b)}{\sigma_{\mathrm{i} n}^{p p}} \times \frac{d \sigma^{p p}}{d M^{2} d y}
$$

where $N_{\text {coll }}(b)$ is the number of binary nucleon nucleon collisions at an impact parameter $b$ calculated using Glauber model [16] and $\sigma_{\mathrm{in}}$ is the inelastic cross section for $p p$ interaction. We have taken $\sigma_{\mathrm{in}}^{p p}=60 \mathrm{mb}$ and $b=3.6 \mathrm{fm}$ corresponding to 0 $50 \%$ centrality at $\sqrt{s_{\mathrm{NN}}}=5.5 \mathrm{TeV}$. The shadowing of PDF's has been taken from [17].

The production of $\tau^{+} \tau^{-}$from a thermally equilibrated system of quarks and gluons can be obtained by convolution of the elementary cross section mentioned above by the thermal distribution of the quarks participating in the process as follows. The number of lepton pairs produced per unit volume per unit time $\left(d N / d^{4} x\right)$ from the annihilation of thermal quarks is given by:

$$
\frac{d N}{d^{4} x}=\int \frac{d^{3} p_{q}}{(2 \pi)^{3}} f_{q}\left(p_{q}\right) \frac{d^{3} p_{\bar{q}}}{(2 \pi)^{3}} f_{\bar{q}}\left(p_{\bar{q}}\right) v_{q \bar{q}} \sigma_{q}
$$

where $f_{q}\left(f_{\bar{q}}\right)$ is the thermal distribution for the quark (anti-quark), $v_{q \bar{q}}$ is the relative velocity between the quark and anti-quark. Making the following change of variables one obtains the distribution of $\tau^{+} \tau^{-}$in terms of the invariant mass and momentum $(P)$ of the pairs,

$$
\frac{d^{3} p_{q}}{E_{q}} \frac{d^{3} p_{\bar{q}}}{E_{\bar{q}}}=\pi \frac{d^{3} P}{E} d M^{2} \sqrt{1-\frac{4 m_{\tau}^{2}}{M^{2}}}
$$

where $m_{\tau}$ is the mass of $\tau, E$ is the energy of the pair. Using Eqs. 6 and 7 and performing the space-time integration over the evolution of the thermal system we get the invariant mass distribution of $\tau^{+} \tau^{-}$at the mid-rapidity.

At multi-TeV energies there could be another contribution to heavy dilepton production by fusion of two gluons. As discussed in Ref [18] the gluon fusion process is via a virtual quark loop and an intermediate $\mathrm{Z}$ or Higgs boson. This process was found to be 

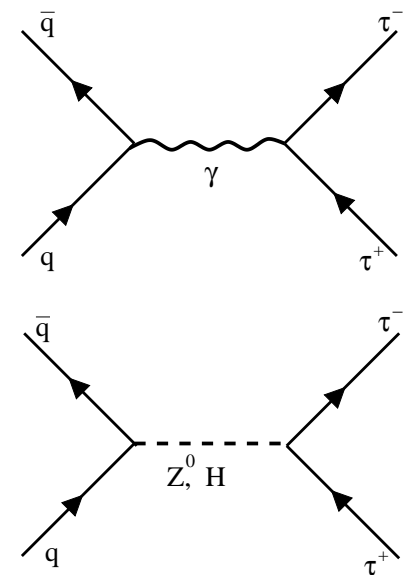

FIG. 1: Feynman diagrams for heavy dilepton production.

dominant for mass of lepton pair greater than the mass of $\mathrm{W}$ boson. Our results are concentrated in the mass range of 4 to $20 \mathrm{GeV}$, where the contribution from such process is negligible.

\section{SPACE-TIME EVOLUTION}

The space time evolution of the system formed in $\mathrm{Pb}+\mathrm{Pb}$ collisions at $\sqrt{s_{\mathrm{NN}}}=5.5 \mathrm{TeV}$ has been studied by using ideal relativistic hydrodynamics [19] with longitudinal boost invariance [20] and cylindrical symmetry. We assume that the system reaches equilibration at a proper time $\tau_{i}=0.08 \mathrm{fm} / \mathrm{c}$ after the collision. The initial temperature, $T_{i}$ is taken to be $700 \mathrm{MeV}$ and is calculated assuming the hadronic multiplicity $(\mathrm{dN} / \mathrm{dy}) \sim 2100[21]$. We use the equation of state (EoS) obtained from the lattice QCD calculations by the MILC collaboration [22] for the partonic phase. For the hadronic phase EoS all the resonances up to mass $2.5 \mathrm{GeV}$ have been considered 23]. The crossover temperature $\left(T_{c}\right)$ between hadronic and partonic phase is taken to be $175 \mathrm{MeV}$ [24].

\section{RESULTS}

Figure 2(a) shows the yield $\left(\frac{d N}{d M d y}\right)$ for $\tau$ lepton pair as a function of $\tau^{+} \tau^{-}$pair invariant mass for $\mathrm{Pb}+\mathrm{Pb}$ collisions at $\sqrt{s_{\mathrm{NN}}}=5.5 \mathrm{TeV}$. The contributions from Drell Yan (DY, dashed line) and thermal partonic medium (QGP, solid line) are shown. The Drell Yan contribution is higher than the thermal contribution for all the mass range studied. The difference seems to increase with increase in $\tau^{+} \tau^{-}$pair mass.
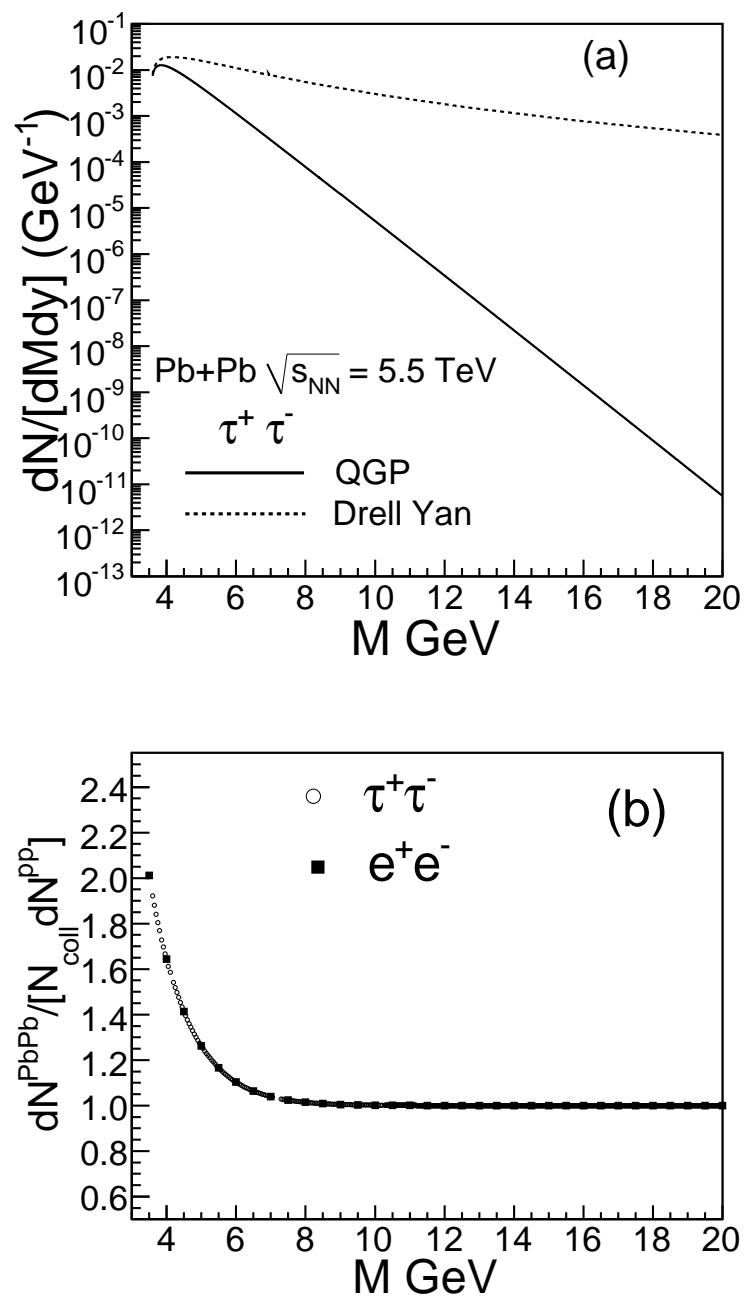

FIG. 2: (a) $\tau$ lepton pair yields as a function of invariant mass of the pair is displayed for $\mathrm{Pb}+\mathrm{Pb}$ collisions at $\sqrt{s_{\mathrm{NN}}}=5.5 \mathrm{TeV}$. Solid line indicates the spectra from quark gluon plasma and the dashed line stands for contribution from DY process. In (b) the ratio $\frac{d N^{P b P b}}{d M d y} /$ $\left[N_{\text {coll }} \frac{d N^{p p}}{d M d y}\right]$ is shown, here $\frac{d N^{P b P b}}{d M d y}$ is the sum of the contribution shown in (a), $\frac{d N^{p p}}{d M d y}$ is the DY contribution from $p p$ collision, and $N_{\text {coll }}=1369$ for $\mathrm{Pb}+\mathrm{Pb}$ collisions at $\sqrt{s_{\mathrm{NN}}}=5.5 \mathrm{TeV}$. The ratio for electron-positron pair is also displayed (solid square).

Figure 2(b) shows the ratio $\frac{d N^{P b P b}}{d M d y} /\left[N_{\text {coll }} \frac{d N^{p p}}{d M d y}\right]$. $\frac{d N^{P b P b}}{d M d y}$ is the sum of the contributions shown in Figure 2(a) from $\mathrm{Pb}+\mathrm{Pb}$ collisions. The quantity $\left[N_{\text {coll }} \frac{d N^{p} p}{d M d y}\right]$ is the number of binary collisions scaled contribution from DY process obtained using Eq. 5 This contribution can be estimated from the measurement in $\mathrm{p}+\mathrm{p}$ collisions at the same energy $(\sqrt{s}$ $=5.5 \mathrm{TeV}$ ). We observe that the ratio is above unity 
for the mass range of 4 to $6 \mathrm{GeV}$. Starting with a value $\sim 2$ at mass of $4 \mathrm{GeV}$ it decreases toward unity beyond mass of $6 \mathrm{GeV}$. This indicates that one should be able to extract a clear information of thermal contribution from partonic source at LHC energies using heavy lepton pair measurement within the mass window of 4 to $6 \mathrm{GeV}$. It is interesting to note that the ratio plotted in Fig. 2 for the heaviest pairs $\left(\tau^{+} \tau^{-}\right)$is very similar to that for the lightest lepton pairs $\left(e^{+} e^{-}\right)$.

In this first such case study, we have not discussed the transverse momentum distribution of $\tau^{+} \tau^{-}$pair, these studies are planned to be presented subsequently. Moreover, we do not discuss the experimental scenario for the measurements of $\tau^{+} \tau^{-}$spectra estimated here for heavy ion collisions at the highest LHC energy. For more details we refer to [25]. The $\tau$ particle is the only lepton heavy enough to decay into hadrons. $\tau$ leptons are considered to be a signature in several discovery channels related to the Standard Model Higgs boson at low masses, the MSSM Higgs boson or Super-symmetry (SUSY). Hence experimental plans exist at LHC to reconstruct them in one-prong (one charged pion) and three-prong (three charged pions) decay topologies. The lifetime of the $\tau$ lepton $(c \tau=87.11 \mu \mathrm{m})$ in principle allows for the reconstruction of its decay vertex in the case of three-prong decays. The flight path in the detector increases with the Lorentz boost of the $\tau$ lepton, but at the same time the angular separation of the decay products decreases. A resulting transverse impact parameter of the $\tau$ decay products can be used to distinguish them from objects originating from the production vertex. In fact experiments at LHC claim the overall efficiency for reconstructing good quality tracks from $\tau$ lepton hadronic decays is of the order of $82 \%$ [25]. Further the excel- lent knowledge of $\tau$ decay modes and detection from low energy experiments 26] makes the heavy lepton pair production measurements feasible at LHC.

\section{SUMMARY}

We have carried out a first case study of $\tau$ lepton pair production for $\mathrm{LHC}, \mathrm{Pb}+\mathrm{Pb}$ collisions at midrapidity for $\sqrt{s_{\mathrm{NN}}}=5.5 \mathrm{TeV}$. The LHC energy is a factor 27 more compared to RHIC, this should allow for significant production of $\tau$ leptons for LHC energies. The main sources for $\tau$ pair production is by quark and anti-quark annihilation mediated through photon, Z and Higgs bosons. The contribution from gluon fusion process via virtual quark loop and intermediate $\mathrm{Z}$ and Higgs boson is negligibly small in the mass range of our calculations ( 4 to $20 \mathrm{GeV}$ ). The Drell Yan contribution is found to be higher than the thermal contribution from partonic sources for the entire mass range studied. The non-thermal contributions could be measured experimentally through $p+p$ collisions, then the ratio of yields from nucleusnucleus collisions to the yields for the binary collision scaled $\mathrm{p}+\mathrm{p}$ collisions is found to be above unity for the mass range of $4-6 \mathrm{GeV}$. This indicates that the invariant mass window for the observation of thermal $\tau$ leptons at LHC energy lies in the domain 4-6 $\mathrm{GeV}$.

\section{Acknowledgments}

BM is supported by DAE-BRNS project Sanction No. 2010/21/15-BRNS/2026. JA is partially supported by DAE-BRNS project Sanction No. 2005/21/5-BRNS/2455. SM would like to thank CSIR for financial support. We thank Dr Zhangbu $\mathrm{Xu}$ for useful discussions.
[1] L. D. McLerran and T. Toimela, Phys. Rev. D 31, 545 (1985).

[2] C. Gale and J.I. Kapusta, Nucl. Phys. B 357, 65 (1991).

[3] H.A. Weldon, Phys. Rev. D 42, 2384 (1990).

[4] J. Alam, S. Raha and B. Sinha, Phys. Rep. 273, 243 (1996).

[5] J. Alam, S. Sarkar, P. Roy, T. Hatsuda and B. Sinha, Ann. Phys. 286, 159 (2001) 159.

[6] R. Rapp and J. Wambach, Adv. Nucl. Phys. 25, 1 (2000).

[7] G.E. Brown and M. Rho, Phys. Rep. 269, 333 (1996).

[8] P. Mohanty, J.K. Nayak, J. Alam and S. K. Das, Phys. Rev. C 82, 034901 (2010); J. K. Nayak and J. Alam, Phys. Rev. C80, 064906 (2009); J. Deng Q. Wang, N. Xu and P. Zhuang, arXiv:1009.3091

[9] P. Mohanty, J. Alam and B. Mohanty,
arXiv:1008.1112

[10] G. Agakichiev et al., CERES Collaboration, Eur. Phys. J. C 41, 475 (2005); D. Adamova et al., CERES Collaboration, Phys. Rev. Lett. 91, 042301 (2003).

[11] R. Arnaldi et al., NA60 Collaboration, Eur. Phys. J. C 59, 607 (2009);Phys. Rev. Lett. 100, 022302 (2008).

[12] A. Adare et al., PHENIX Collaboration, Phys. Rev. C 81, 034911 (2010).

[13] Quarks and Leptons: An Introductory Course in Modern Particle Physics, F. Halzen and A. D. Martin, John Wiley \& Sons, NY 1984.

[14] P. V. Ruuskanen in Quark Gluon Plasma, Edited by R. C. Hwa, World Scientific 1990.

[15] H. L. Lai, J. Huston, S. Kuhlmann, J. Morfin, F. Olness, J. F. Owens, J. Pumplin and W. K. Tung, Eur. Phys. J. C 12, 375 (2000). 
[16] C. Y. Wong, Introduction to High-Energy HeavyIon Collisions, World Scientific, Singapore, 1994.

[17] K.J. Eskola, V.J. Kolhinen and P.V. Ruuskanen, Nucl.Phys. B 535, 351 (1998).

[18] S. S. D. Willenbrock and D. A. Dicus, Phys. Lett. B156, 429 (1985).

[19] H. von Gersdorff, M. Kataja, L. D. McLerran and P. V. Ruskanen, Phys. Rev. D 34, 794 (1986).

[20] J. D. Bjorken, Phys. Rev. D 27, 140 (1983).

[21] N. Armesto, N. Armesto, N. Borghini, S. Jeon, U.A. Wiedemann (ed), J. Phys. G: Nucl. Part. Phys. 35 2008.

[22] C. Bernard et al., Phys. Rev. D 75, 094505 (2007).
[23] B. Mohanty and J. Alam, Phys. Rev. C68, 064903 (2003).

[24] Y. Aoki, Z. Fodor, S. D. Katz and K. K. Szab, Phys. Lett. B 643, 46 (2006).

[25] R. Adolphi et al., CMS Collaboration, JINST 3, S08004 (2008); G. Aad et al., ATLAS Collaboration, JINST 3, S08003 (2008); arXiv:0901.0512 K. Aamodt et al., ALICE Collaboration, JINST 3, S08002 (2008).

[26] S. Schael et al., Phys. Rept., 421, 191 (2005); T. E. Coan et al., Phys. Rev. Lett., 92, 232001 (2004); T. E. Browder et al., Phys. Rev., D 61, 052004 (2000). 